\title{
MicroRNA-200c delivered by solid lipid nanoparticles enhances the effect of paclitaxel on breast cancer stem cell
}

This article was published in the following Dove Press journal:

International Journal of Nanomedicine

12 December 2016

Number of times this article has been viewed

\author{
Jingwen Liu' \\ Tingting Meng' \\ Ming Yuan' \\ Lijuan Wen' \\ Bolin Cheng' \\ $\mathrm{Na}$ Liu' \\ Xuan Huang ${ }^{2}$ \\ Yun Hong ${ }^{3}$ \\ Hong Yuan' \\ Fuqiang $\mathrm{Hu}^{\prime}$ \\ 'Department of Pharmaceutics, \\ College of Pharmaceutical Sciences, \\ Zhejiang University, Hangzhou, \\ ${ }^{2}$ Department of Pharmacy, School of \\ Medicine Science, Jiaxing University, \\ Jiaxing, ${ }^{3}$ The First Affiliated Hospital, \\ College of Medicine, Zhejiang \\ University, Hangzhou, Zhejiang, \\ People's Republic of China
}

Background: One of the major obstacles in the treatment of breast cancer is breast cancer stem cells (BCSC) which are resistant to standard chemotherapeutic drugs. It has been proven that microRNA-200c (miR-200c) can restore sensitivity to microtubule-targeting chemotherapeutic drugs by reducing the expression of class III $\beta$-tubulin. In this study, combination therapy with miR-200c and paclitaxel (PTX) mediated by lipid nanoparticles was investigated as an alternative strategy against BCSC.

Materials and methods: A cationic lipid 1,2-dioleoyl-3-trimethylammonium-propane was strategically selected to formulate solid lipid nanoparticles (SLN) for miR-200c delivery. Nanostructured lipid carriers (NLC) with $20 \mathrm{wt} \%$ oleic acid were prepared for PTX delivery. Mammospheres, which gained the characteristics of BCSC, were used as a cell model to evaluate the efficiency of combination therapy.

Results: The cationic SLN could condense anionic miRNA to form SLN/miRNA complexes via charge interactions and could protect miRNA from degradation by ribonuclease. SLN/miR-200c complexes achieved 11.6-fold expression of miR-200c after incubation for 24 hours, compared with that of Lipofectamine ${ }^{\mathrm{TM}} 2000 / \mathrm{miR}-200 \mathrm{c}$ complexes $(* P<0.05)$. Intracellular drug release assay proved that miRNA can be released from SLN/miRNA complexes efficiently in 12 hours after cellular uptake. After BCSC were transfected with SLN/miR-200c, the expression of class III $\beta$-tubulin was effectively downregulated and the cellular cytotoxicity of PTX-loaded NLC (NLC/PTX) against BCSC was enhanced significantly $(* * P<0.01)$.

Conclusion: The results indicated that the cationic SLN could serve as a promising carrier for miRNA delivery. In addition, the combination therapy of miR-200c and PTX revealed a novel therapeutic strategy for the treatment of BCSC.

Keywords: breast cancer stem cells, microRNA-200c, paclitaxel, solid lipid nanoparticles, nanostructured lipid carriers, combination therapy

\section{Introduction}

Breast cancer is the leading cause of cancer death among females worldwide. ${ }^{1}$ Surgery, chemotherapy, radiotherapy, and hormonal therapy are common therapeutic options for breast cancer patients currently; however, there still remains the issue of tumor recurrence, metastasis, and acquisition of drug resistance..$^{2-4}$ Emerging evidence suggests that breast cancer stem cells (BCSC), which possess the properties of self-renewal and differentiation, may play critical roles. ${ }^{5,6}$ Thus, the eradication of BCSC is an important goal to cure breast cancer. ${ }^{7}$ One of the major obstacles toward treatment of BCSC is their resistance to standard chemotherapeutic drugs. ${ }^{89}$ Therefore, increasing the drug sensitivity is a vital step of improved treatment toward BCSC.
Correspondence: Fuqiang $\mathrm{Hu}$ Department of Pharmaceutics, College of Pharmaceutical Sciences,

Zhejiang University, 866 Yuhangtang

Road, Hangzhou 310058,

People's Republic of China

Tel/fax +86 57l 8820844 I

Email hufq@zju.edu.cn 
MicroRNAs (miRNAs) are a class of small noncoding RNAs of 21-25 nucleotides in length that exert their regulatory effects through posttranscriptional regulation of genes by binding to the $3^{\prime}$-untranslated region of target messenger RNA (mRNA), leading to target mRNA or cleavage translational repression. ${ }^{10}$ The expression of miRNAs is often altered in cancers, particularly in cancer stem cells, affecting self-renewal and proliferation. ${ }^{11}$ Thus, miRNA can be used as therapeutic tools to eradicate cancer stem cells. ${ }^{12,13}$ It has been reported that microRNA-200c (miR-200c) is significantly downregulated in BCSC compared with non-tumorigenic cells. ${ }^{14}$ Restoration of miRNA-200c can increase chemotherapeutic response of microtubule-binding agents by downregulation of class III $\beta$-tubulin (TUBB3). ${ }^{15,16}$ Based on this, drug combinations using miR-200c and paclitaxel (PTX) may open a new window for therapeutic strategy against BCSC.

Delivery of miRNAs is limited by poor cellular uptake, rapid degradation by nucleases, poor endosomal release, and so on. ${ }^{17,18}$ Therefore, the development of safe and efficient miRNA carriers is a prerequisite for the success of combination therapy. Currently, the combination of gene and chemical drugs can be classified into three categories: 1) chemotherapy combined with gene carrying nanoparticles, ${ }^{19}$ 2) codelivery; ${ }^{20}$ and 3) use of multiple nanocarriers. ${ }^{21}$ Using separate delivery systems has the advantage of controlling timing and dosage regimen for the chemo and gene drugs. ${ }^{22}$ Lipid nanoparticles possess many advantages, including biocompatibility, rapid cellular uptake, and potential for large-scale production. ${ }^{23,24}$ In this respect, we selected a cationic lipid 1,2-dioleoyl-3trimethylammonium-propane (DOTAP) to formulate solid lipid nanoparticles (SLN) for miRNA delivery. Nanostructured lipid carriers (NLC) consisting of solid and liquid lipids are a new generation of SLN. NLC have the advantages of improved drug entrapment efficiency (EE) and drug loading (DL) capacity than their SLN counterparts. ${ }^{25}$ Thus, NLC were applied to deliver PTX.

In this study, the physicochemical properties of SLN and NLC obtained were evaluated separately. The human breast cancer (MCF-7) cells were cultured in nonadherent conditions to form mammospheres. The mammospheres, which were proved to be enriched of BCSC by our previous study (Meng, unpublished data, 2016), were used as an in vitro model to study the combination therapy effect. As TUBB3 is a direct target of miR-200c, real-time polymerase chain reaction (PCR) and Western blot were performed to examine the gene and protein expression of TUBB3 after miR-200c transfection. The cytotoxicity of PTX-loaded NLC after transfection was investigated to verify whether miR-200c delivered by SLN could enhance the therapeutic effects of PTX against BCSC.

\section{Materials and methods Materials}

Monostearin was purchased from Shanghai Chemical Reagent Co. Ltd. (Shanghai, People's Republic of China). DOTAP was obtained from Lipoid GmbH (Ludwigshafen, Germany). Oleic acid was supplied by Shuanglin Chemical Industry Co. Ltd. (Hangzhou, People's Republic of China). Octadecanoic acid was purchased from Fluka (Sigma-Aldrich, St Louis, MO, USA). Polyvinyl alcohol (PVA 0486) was obtained from Beijing Chemicals Co. Ltd. (Beijing, People's Republic of China). Poloxamer 188 was supplied by Shenyang Jiqi Pharmaceutical Co. Ltd. (Shenyang, People's Republic of China). Tris-base was purchased from Sigma-Aldrich Co. (St Louis, MO, USA). Agarose G-10 was obtained from Biowest (Granada, Spain). PTX was purchased from Shanghai Zhongxi Sunve (Shanghai, People's Republic of China). Taxol was obtained from BristolMyers Squibb (New York, NY, USA). Lipofectamine ${ }^{\mathrm{TM}}$ 2000 was obtained from Thermo Fisher Scientific (Waltham, MA, USA). miR-200c mimics (miR-200c mimics sequence: 5'-UAAUACUGCCGGGUAAUGAUGGA-3'), negative control miRNA, CY3-labeled miRNA (miRNA-CY3), and fluorescein amidite (FAM)-labeled miRNA (miRNA-FAM) were supplied by GenePharma (Shanghai, People's Republic of China). Fluorescein isothiocyanate (FITC) was purchased from Acros Organic (Fair Lawn, NJ, USA). 4-Nitrophenyl phosphate disodium was purchased from Sigma-Aldrich. Dulbecco's Modified Eagle's Medium (DMEM)/F12 1:1 and B27 were purchased from HyClone (Logan, UT, USA). Human recombination epidermal growth factor and human recombination b-fibroblast growth factor were supplied by Perpro Tech Inc. (Rocky Hill, NJ, USA). Insulin was supplied by Sigma-Aldrich Co. B-27 Supplement was obtained from Thermo Fisher Scientific. The bicinchoninic acid (BCA) protein assay kit was purchased from the Beyotime (Shanghai, People's Republic of China). All other chemicals were analytical reagent grade.

\section{Preparation of SLN and SLN/miRNA complexes}

SLN with $0 \mathrm{wt} \%, 10 \mathrm{wt} \%, 20 \mathrm{wt} \%$, and $30 \mathrm{wt} \%$ content of DOTAP were prepared by aqueous solvent diffusion method reported in our previous study. ${ }^{25}$ Briefly, $30 \mathrm{mg}$ monostearin was dissolved in $3 \mathrm{~mL}$ ethanol, and then the monostearin was melted at $50^{\circ} \mathrm{C}$. Amounts of 3, 6, and $9 \mathrm{mg}$ of DOTAP were used instead of the same amount of monostearin to 
prepare SLN with $10 \mathrm{wt} \%, 20 \mathrm{wt} \%$, and $30 \mathrm{wt} \%$ content of DOTAP, respectively. The resulting organic solution was then dispersed rapidly into $30 \mathrm{~mL}$ poloxamer 188 solution $(0.1 \%, \mathrm{w} / \mathrm{v})$ agitating (DC-40, Hangzhou Electrical Engineering Instruments, People's Republic of China) at $400 \mathrm{rpm}$ in $50^{\circ} \mathrm{C}$ water bath for 5 minutes. Then, the solution was gradually cooled to the room temperature.

For preparation of SLN/miRNA complexes, miRNA solution was added to SLN colloidal solution, and the mixture was incubated for 30 minutes at room temperature.

\section{Preparation of NLC and PTX-loaded NLC}

NLC and PTX-loaded NLC (NLC/PTX) were prepared by aqueous solvent diffusion method. An amount of $12 \mathrm{mg}$ monostearin, $12 \mathrm{mg}$ soybean phosphatidylcholine (S100), and $6 \mathrm{mg}$ oleic acid were used as lipid materials and dissolved in $3 \mathrm{~mL}$ ethanol at $50^{\circ} \mathrm{C}$. The melted solution was dispersed into $30 \mathrm{~mL}$ polyvinyl alcohol solution $(1 \%$, w/v) agitating at $400 \mathrm{rpm}$ in $50^{\circ} \mathrm{C}$ water bath for 5 minutes. The $\mathrm{pH}$ value of the NLC dispersion was adjusted by addition of $0.1 \mathrm{M}$ acetic acid to form nanoparticle aggregation. NLC were collected by centrifugation $(20,000 \mathrm{rpm}, 10$ minutes $)$. The NLC precipitate was then resuspended in $30 \mathrm{~mL}$ polyvinyl alcohol solution $(1 \%, \mathrm{w} / \mathrm{v})$. To prepare NLC/PTX, the same amount of lipid materials with $1.5 \mathrm{mg}$ PTX was mixed, and then followed the method described earlier.

FITC-labeled octadecanoic acid was synthesized according to our previously reported study ${ }^{24}$ and was incorporated into the lipid matrix to formulate fluorescence SLN or NLC.

\section{Characterization of the nanoparticles}

The average diameter and zeta potential of nanoparticles were measured by dynamic light scattering using a zetasizer (Nano-ZS90; Malvern Instruments, Malvern, UK).

The morphology of nanoparticles was observed by transmission electron microscopy (JEM-1230EX; JEOL, Tokyo, Japan). Specimens were prepared by dripping suspensions onto copper grids, followed by negative staining with $2 \%$ aqueous solution of sodium phosphotungstate.

\section{Gel retardation assay}

The capability of cationic SLN to condense miRNA (negative control miRNA) was evaluated on agarose gel electrophoresis $(1 \%, \mathrm{w} / \mathrm{v})$ under a current voltage of $120 \mathrm{~V}$ for 25 minutes in a buffer solution (40 mM Tris- $\mathrm{HCl}, 1 \%$, v/v acetic acid, $1 \mathrm{mM}$ ethylenediaminetetraacetic acid). SLN/miRNA nanoparticles containing $0.13 \mu \mathrm{g}$ of miRNA were prepared at different weight ratios by varying the concentration of SLN. Images were obtained using an ultraviolet transilluminator and a digital imaging system (GL 200; Kodak, Windsor, $\mathrm{CO}, \mathrm{USA})$.

\section{RNase A protection assay}

An amount of $2 \mu \mathrm{L}$ of $10 \mathrm{mg} / \mathrm{mL}$ RNase A was added to SLN/miRNA complexes (90:1, w/w, $0.13 \mu \mathrm{g}$ miRNA/well), and the mixtures were incubated at $37^{\circ} \mathrm{C}$ for 30 minutes. An amount of $0.5 \mathrm{M}$ ethylenediaminetetraacetic acid was used to terminate the reaction. Subsequently, $10 \%$ heparin sodium solution was added to release miRNA from nanoparticles. The final samples were further incubated in an incubator shaker $\left(60 \mathrm{rpm}, 37^{\circ} \mathrm{C}\right)$, and electrophoresis was performed with $1 \%$ agarose gel for 25 minutes at $120 \mathrm{~V}$. The gel was then observed by ultraviolet transilluminator and a digital imaging system.

\section{Drug encapsulation efficiency and DL}

The PTX contents were detected by high-performance liquid chromatography (HPLC; Agilent Technologies, Santa Clara, CA, USA). The mobile phase was acetonitrile-water (45:55, $\mathrm{v} / \mathrm{v}$ ) at a rate of $1.0 \mathrm{~mL} / \mathrm{min}$ at $35^{\circ} \mathrm{C}$. The ultraviolet detector was set to $227 \mathrm{~nm}$.

The NLC/PTX was precipitated by adding $0.1 \mathrm{M}$ acetic acid, and then the supernatant was collected after centrifugation. The drug content in the obtained supernatant was measured by HPLC as described earlier. The drug EE and DL of SLN values were calculated by the following equations:

$$
\begin{gathered}
\mathrm{EE}=\left(\mathrm{W}_{\mathrm{d}}-\mathrm{W}_{\mathrm{S}}\right) / \mathrm{W}_{\mathrm{d}} \times 100 \% \\
\mathrm{DL}=\left(\mathrm{W}_{\mathrm{d}}-\mathrm{W}_{\mathrm{S}}\right) /\left(\mathrm{W}_{\mathrm{d}}-\mathrm{W}_{\mathrm{S}}+\mathrm{W}_{\mathrm{L}}\right) \times 100 \%
\end{gathered}
$$

where $\mathrm{W}_{\mathrm{d}}$ is the weight of drug added in the system, $\mathrm{W}_{\mathrm{S}}$ is the analyzed weight of drug in supernatant after centrifugation, and $\mathrm{W}_{\mathrm{L}}$ is the weight of lipid added in the system.

\section{In vitro release test}

miRNA-FAM was used to form complexes with different weight ratios of SLN to miRNA-FAM. Then, the SLN/ miRNA-FAM complexes were suspended in Eppendorf tubes containing Tris-base ( $\mathrm{pH} 7.4)$. The tubes were placed in an incubator shaker $\left(60 \mathrm{rpm}, 37^{\circ} \mathrm{C}\right)$. At predetermined time intervals, the suspensions were centrifuged at 20,000 rpm for 10 minutes. The amount of miRNA-FAM released was analyzed by fluorescence spectrophotometer with an 
excitation wavelength of $480 \mathrm{~nm}$ and an emission wavelength of $520 \mathrm{~nm}$.

To study in vitro release profiles of PTX-loaded NLC, the precipitate of drug-loaded nanoparticles were redispersed in phosphate-buffered saline (PBS) $(\mathrm{pH} 7.4)$ containing $1 \mathrm{M}$ sodium salicylate. The sodium salicylate solution can increase the aqueous PTX solubility. Therefore, it was used to provide a good sink condition for PTX. And then the release medium was shaken horizontally ( $\left.60 \mathrm{rpm}, 37^{\circ} \mathrm{C}\right)$ using an incubator shaker. At predetermined time intervals, $1 \mathrm{~mL}$ of the suspensions was collected and then ultrafiltered/centrifuged. The filters were determined by HPLC as described earlier. The diffusion profile of pure drug dispersion was performed as a comparison.

\section{BCSC sphere culture}

Primary cell culture

MCF-7 cells were cultured in DMEM medium containing fetal bovine serum $(10 \%, \mathrm{v} / \mathrm{v})$, penicillin $(100 \mathrm{U} / \mathrm{mL})$, and streptomycin $(100 \mathrm{U} / \mathrm{mL})$, and maintained in a humidified $37^{\circ} \mathrm{C}$ incubator with $5 \% \mathrm{CO}_{2}$.

\section{Formation of mammospheres}

The serum-free medium (SFM) consisted of human recombinant epidermal growth factor $(20 \mathrm{ng} / \mathrm{mL})$, human recombinant basic fibroblast growth factor $(20 \mathrm{ng} / \mathrm{mL})$, insulin $(5 \mu \mathrm{g} / \mathrm{mL})$, and B27 supplement $(2 \%, \mathrm{v} / \mathrm{v})$. The single MCF-7 cells were suspended in SFM at a density of 5,000 cells $/ \mathrm{mL}$. The medium was added every 5 days. On the fourth, seventh, and ninth day post-cell seeding, images of mammospheres were taken by an inverted microscope.

\section{Real-time PCR quantification of miR-200c and mRNA}

The mammospheres were treated with miR-200c, SLN/ miR-200c, and Lipofectamine ${ }^{\text {TM }}$ 2000/miR-200c. Untreated cell spheroids were used as a blank control. After 24 hours, the mammospheres were collected, washed with PBS, and then treated with TRIzol reagent (Thermo Fisher Scientific). Total RNA was extracted by adding chloroform, further purified by isopropanol precipitation. An amount of $500 \mathrm{ng}$ RNA was first reverse transcribed into cDNA with the sequence-specific stem-loop RT primers for miR-200c (ShenGong, Shanghai, People's Republic of China). Primers for amplification were as follows: 5'-GTCGTATCCAGTGCAGGGTCCGAGG TATTCGCACTGGATACGACTCCATC-3' (stem-loop primer), 5'-GCGGCGGTAATACTGCCGGG-3' (forward primer), and 5'-ATCCAGTGCAGGGTCCGAGG-3' (reverse primer). The real-time PCR amplification of cDNA was then performed using SYBR Green PCR Master Mix (Takara, Shiga, Japan) on the Step one plus system (Thermo Fisher Scientific). The PCR reaction was conducted at $95^{\circ} \mathrm{C}$ for 3 minutes, 40 cycles of $95^{\circ} \mathrm{C}$ for 12 seconds, and $62^{\circ} \mathrm{C}$ for 40 seconds. The miR-200c expression was then analyzed by the $\Delta \Delta \mathrm{CT}$ method and normalized to U6 small nuclear RNA (snRNA) (GenePharma), which was the endogenous control in the corresponding samples.

To measure TUBB3 expression at the mRNA level, 1 $\mu \mathrm{g}$ RNA was transcribed into cDNA. The resulting cDNA was amplified by real time polymerase chain reaction (RTPCR). Primers for amplification of TUBB3 were as follows: 5'-AGTATCCCGACCGCATCA-3' (forward primer) and 5'-CATCCGTGTTCTCCACCAG-3' (reverse primer). The PCR reaction was conducted at $95^{\circ} \mathrm{C}$ for 30 seconds, 40 cycles at $95^{\circ} \mathrm{C}$ for 5 seconds, and $60^{\circ} \mathrm{C}$ for 30 seconds. The TUBB3 gene expression was analyzed by the $\Delta \Delta C T$ method, and normalized to GAPDH, which was the endogenous reference in the corresponding samples.

\section{Intracellular drug release of SLN/miRNA complexes}

To investigate the intracellular drug release of SLN/ miRNA complexes, FITC-labeled SLN (FITC-SLN), and miRNA-CY3 were used to prepare fluorescent complexes. Mammospheres were treated with Accutase-Enzyme Cell Detachment Medium to obtain single cell suspension, and then single cells were seeded at a density of 50,000 cells per well in 24-well plate and cultured in growth medium (DMEM, 10\% fetal bovine) for 12 hours to attach. FITCSLN/miRNA-CY3 was added to the culture medium. After incubation for 4 hours, the culture medium was removed, the cells were rinsed twice with PBS, and fresh medium was added. Further incubated for 0,4 , and 12 hours, the cells were washed with PBS and observed by confocal laser scanning microscope (CLSM, Ix81-FV1000; Olympus Corporation, Tokyo, Japan).

\section{Western blot assay}

After transfection for 48 hours, the cell spheres were collected and washed with PBS, then incubated with the radioimmunoprecipitation assay (RIPA) lysis buffer (Shenggong, Shanghai, People's Republic of China) containing a protease inhibitor on ice for 15 minutes. The cell lysate was centrifuged at $4^{\circ} \mathrm{C}(10,806 \mathrm{rpm}, 5$ minutes $)$. The supernatant was collected and the total protein concentration was determined using BCA protein assay kit. An amount of $20 \mu \mathrm{g}$ protein of each sample was separated on $8 \%$ sodium dodecyl sulfate polyacrylamide gel electrophoresis gels and transferred to 
nitrocellulose membranes, and then blocked with 5\% skim milk in PBS containing 0.1\% Tween 20 (T-PBS buffer) for 1 hour. After the membrane was washed with T-PBS, the primary antibodies of TUBB3 and $\beta$-tubulin diluted in T-PBS were added and incubated for 4 hours at room temperature, respectively. After incubation with appropriate secondary antibody, signals were detected via an enhanced chemiluminescence detection system named enhanced chemiluminescence (ECL) (Shenggong).

\section{Cellular uptake of fluorescent NLC}

Mammospheres were treated with FITC-labeled NLC for 12 and 24 hours. Then, the cells were washed twice with PBS (pH 7.4) and observed by CLSM with a $40 \times$ water immersion objective. Excitation wavelength and emission wavelength were 488 and $520 \mathrm{~nm}$, respectively. Stacked images were obtained by piling up the images scanned layer by layer. Each scanning layer was $1.2 \mu \mathrm{m}$ in thickness.

\section{In vitro cytotoxicity}

The in vitro anticancer activity of combined gene-chemo therapy was evaluated by a cell viability assay. Mammospheres grown in 96-well plates were incubated with naked miR-200c, SLN/miRNA negative control (SLN/miRNA NC), SLN/miR-200c, and Lipofectamine ${ }^{\text {TM }}$ 2000/miR-200c (equal miRNA concentration) for 24 hours. Untreated cell spheroids were used as a blank control. Afterward, all groups were treated with taxol or PTX-loaded NLC with different concentration. Cells were further incubated for 48 hours. The percentage of the viable cells was measured by an acid phosphatase assay. The plates were centrifuged (10 minutes, $400 \times g$ ) to spin down the spheroids. The supernatant was discarded and the cells were washed with PBS. Centrifugation was repeated, and PBS was added to the supernatant to reach a final volume of $100 \mu \mathrm{L}$. Then, $100 \mu \mathrm{L}$ of the assay buffer ( $0.1 \mathrm{M}$ sodium acetate, $0.1 \%$ Triton-X-100, $2 \mathrm{mg} / \mathrm{mL}$ of paranitrophenylphosphate) was added per well and incubated for 90 minutes at $37^{\circ} \mathrm{C}$. Ten microliters of $1 \mathrm{~mol} / \mathrm{L} \mathrm{NaOH}$ solution was added to each well to terminate the reaction. The absorbances of each well was measured at $405 \mathrm{~nm}$ using a microplate reader (SpectraMax M5; Molecular Devices LLC, Sunnyvale, CA, USA).

\section{Statistical analysis}

The results were expressed as mean \pm standard deviation. Statistical significance was analyzed using $t$-test. The resultant $P$-value was expressed as $* P<0.05$ and $* * P<0.01$. Differences were considered to be statistically significant if $P$-value was $<0.05$.
Table I The average $d_{n}, \zeta$, and PI of SLN containing different amount of DOTAP

\begin{tabular}{llll}
\hline DOTAP ratio $(w t \%)$ & $\mathbf{d}_{\mathbf{n}}(\mathbf{n m})$ & $\mathbf{Z}(\mathbf{m V})$ & $\mathbf{P I}$ \\
\hline 0 & $65.9 \pm 13.2$ & $-19.7 \pm 0.7$ & $0.18 \pm 0.01$ \\
10 & $48.0 \pm 10.5$ & $3.9 \pm 1.0$ & $0.30 \pm 0.02$ \\
20 & $28.4 \pm 4.2$ & $18.4 \pm 1.3$ & $0.24 \pm 0.01$ \\
30 & $31.2 \pm 2.6$ & $18.7 \pm 1.0$ & $0.34 \pm 0.06$ \\
\hline
\end{tabular}

Note: Data presented as mean \pm standard deviation.

Abbreviations: DOTAP, I,2-dioleoyl-3-trimethylammonium-propane; $d_{n}$, diameter; $\mathrm{PI}$, polydispersity index; SLN, solid lipid nanoparticles; $\zeta$, zeta potential.

\section{Results and discussion Characterization of SLN and NLC} SLN, NLC, and PTX-loaded NLC (NLC/PTX) were prepared by solvent diffusion method as reported in our previous work. ${ }^{25} \mathrm{SLN} / \mathrm{miRNA}$ nanoparticles were prepared by mixing an appropriate amount of SLN and miRNAs at room temperature.

Number average diameters and zeta potential of SLN containing different amount of DOTAP are listed in Table 1. SLN showed a decrease in size distribution after adding cationic lipid DOTAP. It was probably attributed to the amphipathy of DOTAP. ${ }^{26}$ The incorporation of DOTAP could enhance the hydrophilicity of SLN surface and decrease the surface tension. As a result, the sizes of SLN incorporated with DOTAP were smaller than that of SLN without DOTAP. However, the physicochemical characterizations of SLN containing 20\% and $30 \%$ DOTAP were slightly different. In this study, SLN containing $20 \mathrm{wt} \%$ DOTAP were selected for further investigation.

The results of physicochemical properties of SLN/miRNA nanoparticles with different weight ratios of SLN to miRNA are summarized in Table 2. When the weight ratio of SLN to miRNA was $60: 1$, the average size increased to $141.8 \pm 3.9 \mathrm{~nm}$ and the zeta potential was $-1.6 \pm 0.9 \mathrm{mV}$. The larger size was probably because the nanoparticles with this weight ratio were in an unstable state and easily aggregated.

The mean particle sizes of NLC and NLC/PTX were $56.1 \pm 9.0$ and $64.5 \pm 3.3 \mathrm{~nm}$, respectively. The zeta potential of NLC and NLC/PTX was $-17.1 \pm 0.2$ and $-16.4 \pm 0.3$, respectively. EE and DL of NLC/PTX were $73.33 \% \pm 2.03 \%$ and $3.51 \% \pm 0.07 \%$, respectively.

Table 2 The average $d_{n}, \zeta$, and PI of SLN/miRNA complexes containing different amount of SLN to miRNA

\begin{tabular}{llll}
\hline SLN/miRNA $(w / w)$ & $d_{n}(\mathrm{~nm})$ & $\zeta(\mathrm{mV})$ & PI \\
\hline $60: 1$ & $141.8 \pm 3.9$ & $-1.6 \pm 0.9$ & $0.51 \pm 0.17$ \\
$90: 1$ & $36.1 \pm 3.0$ & $14.7 \pm 0.9$ & $0.49 \pm 0.14$ \\
$120: 1$ & $48.4 \pm 4.0$ & $15.5 \pm 0.3$ & $0.41 \pm 0.03$ \\
\hline
\end{tabular}

Note: Data presented as mean \pm standard deviation.

Abbreviations: $d_{n}$, diameter; PI, polydispersity index; SLN, solid lipid nanoparticles; $\zeta$, zeta potential. 
Transmission electron microscopy was used to investigate the morphology of SLN, SLN/miRNA, NLC, and NLC/PTX. The sizes of nanoparticles, which are shown in transmission electron microscopy pictures (Figure 1), were consistent with the results measured by Zetasizer. The pictures exhibited that all the nanoparticles had spherical-like shapes with smooth surfaces.

\section{Gel retardation assay}

The ability of the cationic SLN to bind miRNA was investigated by agarose gel electrophoresis. Gradual miRNA retardation was observed when the weight ratio of SLN to miRNA in the complexes increased. When the weight ratio was above $30, \mathrm{SLN} / \mathrm{miRNA}$ nanoparticles were retarded completely (Figure 2A).

\section{RNase A protection assay}

The miRNAs are labile in serum; accordingly, it is important to protect them from degradation and maintain their stability. ${ }^{27}$ In this study, RNase A was used to evaluate the stability of miRNA in SLN/miRNAs complexes. The result is shown in Figure 2B. Compared with the lane of free miRNA treated without RNase A, no band was observed from the lane of free miRNA treated with RNase A. In contrast, clear bands could be found from the lanes of SLN/miRNA, which demonstrated that SLN could effectively protect miRNA from degradation by ribonuclease.

\section{In vitro release test}

The in vitro release study of miRNA from SLN/miRNA was carried out in Tris-base ( $\mathrm{pH} 7.4)$. The calibration curve for the quantification of miRNA-FAM was linear over the range of standard concentration of miRNA-FAM at $1-300 \mathrm{nmol} / \mathrm{L}$ with a correlation coefficient of $R^{2}=0.9996$. Release profiles of SLN/miRNA complexes with different weight ratios are illustrated in Figure 3A. When the complexes were prepared at a weight ratio of 60 , a cumulative release of $48.24 \%$ was observed in 4 hours and $95.64 \%$ in 12 hours. At a weight ratio of 90 , the miRNA was released at $32.71 \%$ in 4 hours and $87.30 \%$ in 12 hours. A higher weight ratio of SLN to miRNA led to a slower release of miRNA from the complexes. At a weight ratio of 120 , miRNA was released at a much slower rate, with the release of $16.25 \%$ for 4 hours and $45.05 \%$ for 12 hours. The results suggested that the release rate of miRNA could be adjusted by varying the charge ratio of SLN to miRNA.

The release rate of PTX from NLC/PTX was measured by HPLC (Figure 3B). The calibration curve for the quantification of PTX was linear over the range of standard concentration of PTX at $0.1-100 \mu \mathrm{g} / \mathrm{mL}$ with a correlation coefficient of $R^{2}=0.9998$. The limitation of detection was $0.01 \mu \mathrm{g} / \mathrm{mL}$. A burst drug release was found at the initial 8 hours. After that, the drug was released relatively slowly. This result was consistent with our previous study. ${ }^{28}$ The distribution of liquid lipid in nanoparticles matrix probably contributed
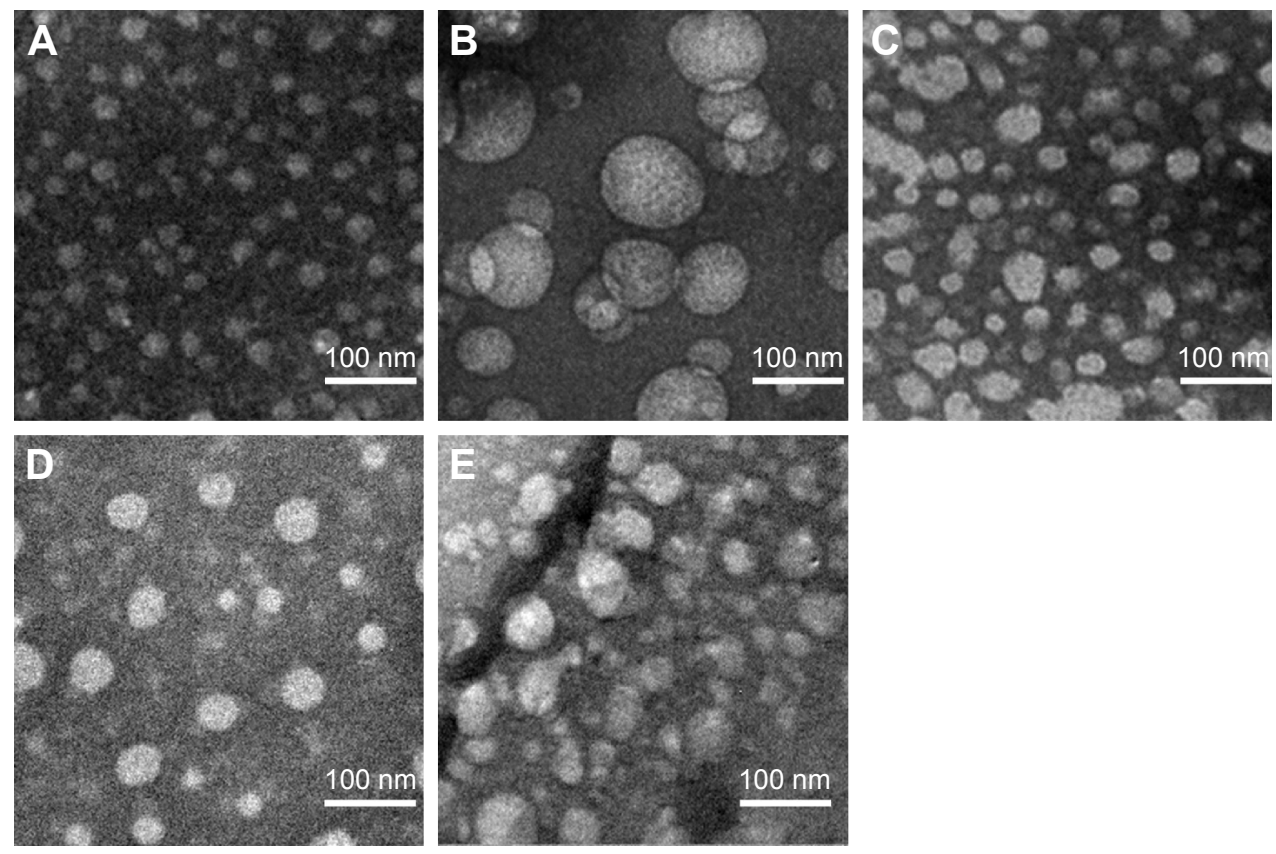

Figure I Transmission electron microscopic images of (A) SLN with 20 wt\% DOTAP, (B) SLN/miRNA (SLN containing 20 wt\% DOTAP, 60:I, w/w), (C) SLN/miRNA (SLN containing 20 wt\% DOTAP, 90:I, w/w), (D) NLC, and (E) NLC/PTX. Magnification is 100,000x.

Abbreviations: DOTAP, I,2-dioleoyl-3-trimethylammonium-propane; NLC, nanostructured lipid nanoparticles; PTX, paclitaxel; SLN, solid lipid nanoparticles. 


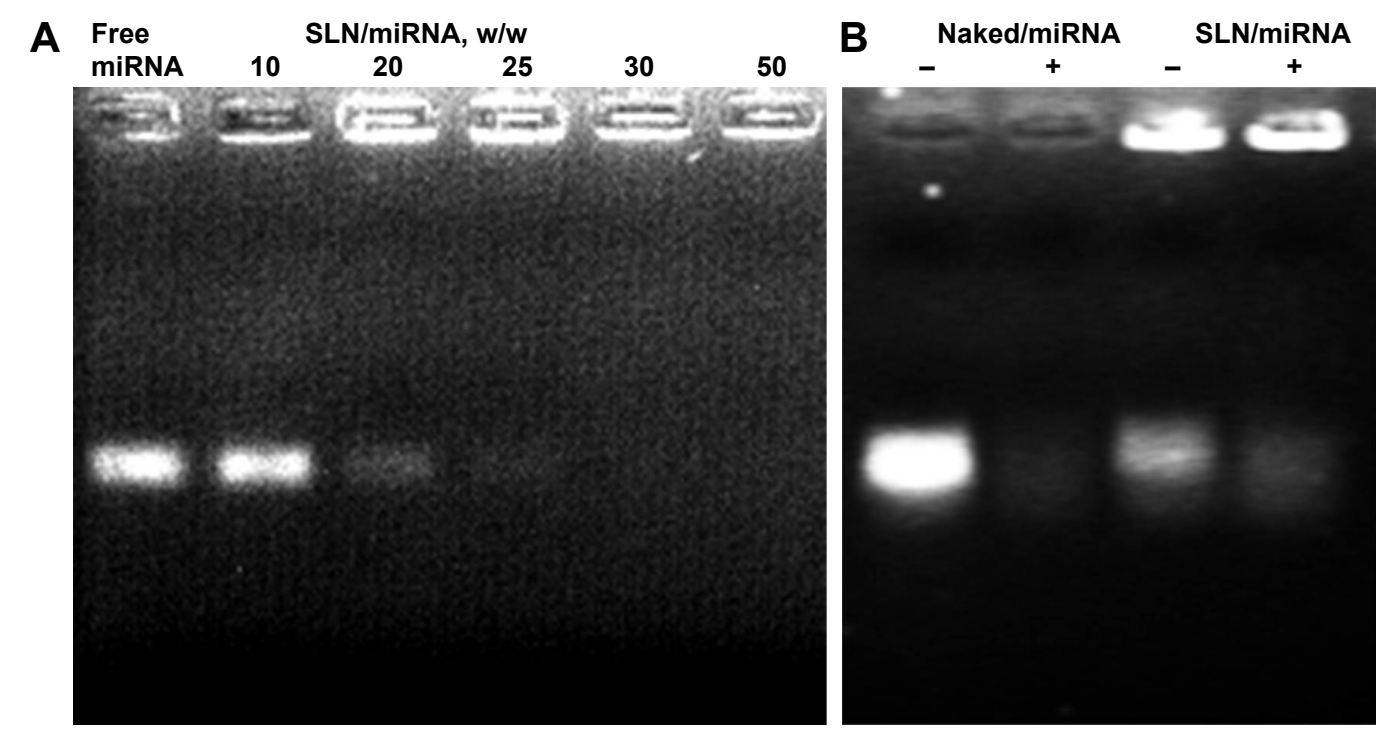

Figure 2 Gel retardation analyses (A) of SLN/miRNA complexes prepared at different weight ratios and RNase A protection assay (B) of SLN/miRNA complexes. Notes: In (B), lanes I-4 represent naked miRNA, naked miRNA + RNase, SLN/miRNA, and SLN/miRNA + RNase, respectively. SLN/miRNA complexes were prepared at the weight ratio of 90 .

Abbreviation: SLN, solid lipid nanoparticles.

to the drug release profiles. Drugs were mostly incorporated into the liquid lipid, which was mostly located at the shell of nanoparticles instead of the core. As a result, the drugs could be released easily through the drug diffusion and the matrix erosion manners in initial hours and exhibited a sustained release afterward.

\section{Formulation of mammosphere}

Mammosphere-forming culture condition has been proven to be an efficient way to enrich tumor stem cells. ${ }^{29}$ Our previous work confirmed that MCF-7 cells under this condition of cultivation had gained the characteristics as BCSC. The percentage of $\mathrm{CD} 44^{+} / \mathrm{CD} 24^{-}$expression, which is a cancer stem cell marker, increased to $36.51 \%$.

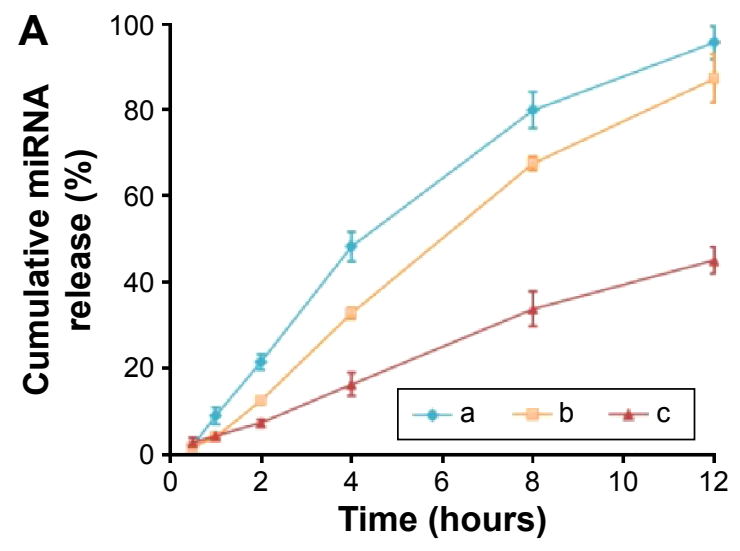

The morphology of mammospheres was observed on an inverted microscope during spheroid growth. As shown in Figure 4, breast cancer cells (MCF-7) grown in SFM resulted in the formation of cellular spheroids. Mammospheres were packed tightly and were $\sim 100 \mu \mathrm{m}$ in diameter by 7 days of incubation. After 9 days under SFM culture conditions, the mammospheres exhibited a compact, rounded shape and attained a diameter of 100-200 $\mu \mathrm{m}$. The seventh day was considered as a treatment initiation point for further study.

\section{Cellular uptake of SLN/miRNA complexes}

To investigate the cellular uptake of SLN/miR-200c complexes, the miR-200c expression after transfection

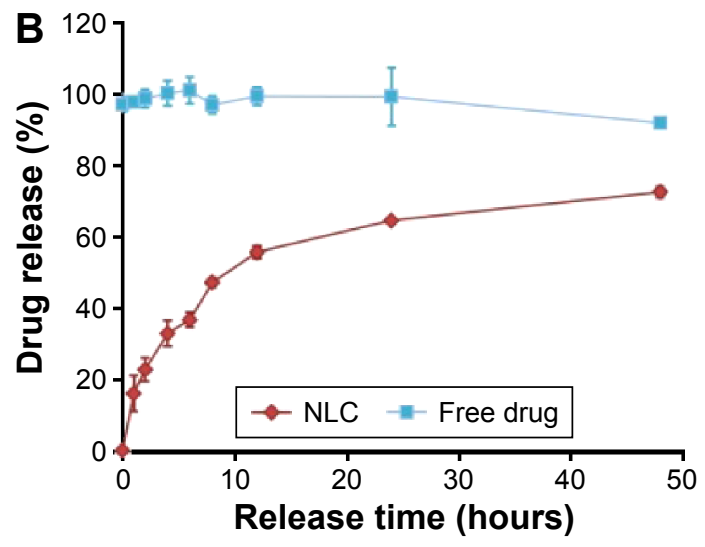

Figure 3 Release profiles (A) of miRNA from SLN/miRNA complexes with different weight ratios in Tris-base ( $n=3$ ). Lines a, b, and c represent SLN/miRNA nanoparticles at the weight ratio of 60:I, 90:I, and I20:I, respectively. Release profiles (B) of PTX from NLC/PTX. The error bars in the graphs represent the standard deviations ( $\mathrm{n}=3$ ). Abbreviations: NLC, nanostructured lipid nanoparticles; PTX, paclitaxel; SLN, solid lipid nanoparticles; miRNA, microRNA. 
Day 4

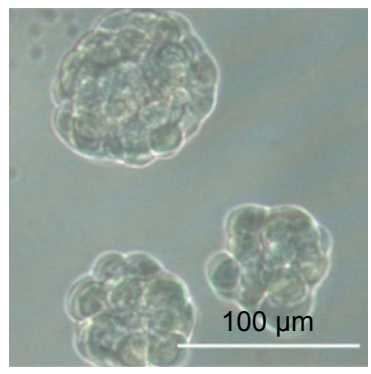

Day 7

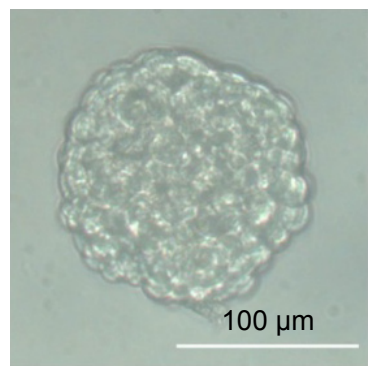

Day 9

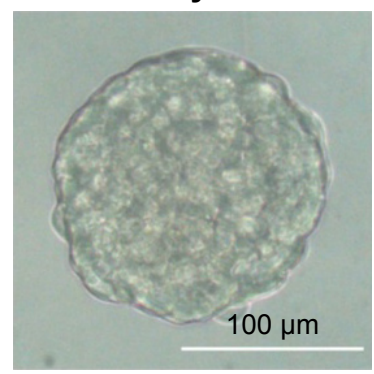

Figure 4 Light microscope images of mammospheres after incubation for 4, 7, and 9 days, respectively. Note: Scale bar: $100 \mu \mathrm{m}$, magnification: $200 \times$.

was evaluated. From Figure 5, naked miR-200c only had 1.95-fold expression of miR-200c over blank group, which demonstrated that miR-200c cannot be delivered efficiently without suitable gene carriers. Compared with the commercial transfection agent Lipofectamine ${ }^{\mathrm{TM}} 2000$, SLN achieved an 11.6-fold expression of miR-200c. This result suggested that SLN had a relatively higher cellular uptake efficiency against mammospheres, indicating its great potential for efficient miRNA delivery.

\section{Intracellular release of SLN/miR-200c complexes}

As only free miRNA could interact with target mRNA in cytoplasm and exert its pharmacological action, the intracellular drug release behaviors of the SLN/miRNA complexes

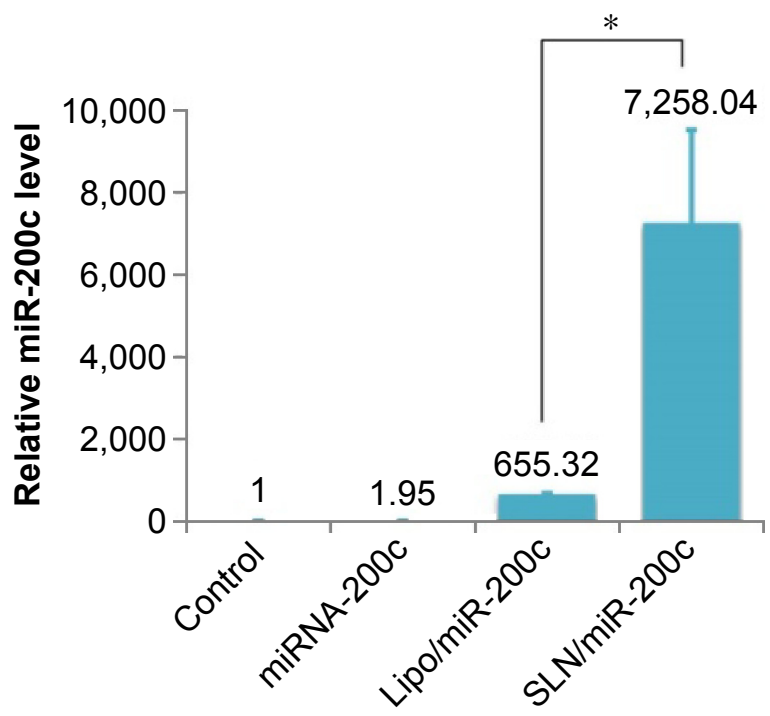

Figure 5 Mammospheres were treated with miR-200c, SLN/miR-200c, and Lipofectamine ${ }^{T M}$ 2000/miR-200c, respectively.

Notes: Untreated spheres were the blank control. After 24 hours, RNA was harvested and miR-200c levels were determined by real-time PCR $(* P<0.05$, compared with Lipofectamine ${ }^{T M}$ 2000/miR-200c group).

Abbreviations: PCR, polymerase chain reaction; SLN, solid lipid nanoparticles; Lipo, Lipofectamine ${ }^{\mathrm{TM}} 2000$. against single-layer BCSC were investigated. FITC-SLN and miRNA-CY3 were used to prepare fluorescent SLN/ miRNA complexes. The images were obtained from CLSM and the results of colocalization of SLN with miRNA were calculated by MetaMorph software.

After cells were incubated with FITC-SLN/miRNA-CY3 for 4 hours, the culture medium was removed and replaced with fresh medium. Figure 6A shows the colocalization of SLN with miRNA in cells after further incubation for different time periods. The overlap of red fluorescence and green fluorescence was decreased gradually with time. Figure $6 \mathrm{~B}$ shows that from 0 to 12 hours, the area a (FITC-SLN) overlap b (miRNA-CY3) decreased from $92 \%$ to $13 \%$ and the area b overlap a decreased from $85 \%$ to $21 \%$, indicating that SLN/miRNA complexes can effectively release miRNA over time.

\section{The TUBB3 mRNA and protein expression}

TUBB3 is a direct target of miR-200c. ${ }^{15}$ We used realtime PCR to investigate the effect of SLN/miR-200c complexes on TUBB3 mRNA expression in mammospheres. Compared with blank control group, SLN/miR-200c and Lipofectamine $^{\mathrm{TM}}$ 2000/miR-200c complexes almost did not influence TUBB3 expression in mRNA level (Figure 7A). As the miRNA can direct the cleavage of target mRNAs or negatively regulate target expression at the transcription level, ${ }^{30}$ we performed Western blot to examine the protein expression of TUBB3. As it shown in Figure 7B, SLN/miR-200c significantly decreased TUBB3 protein expression, and the same effect was found in the Lipofectamine ${ }^{\mathrm{TM}} 2000 /$ miR-200c-treated group.

The decrease of TUBB3 mRNA was not observed after transfection with SLN/miR-200c. However, an obvious decrease in TUBB3 protein level was found, which indicated that miR-200c may affect the expression of TUBB3 primarily through inhibition of TUBB3 translation. 
A
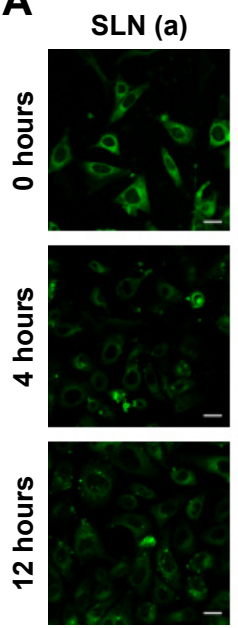

miRNA (b)
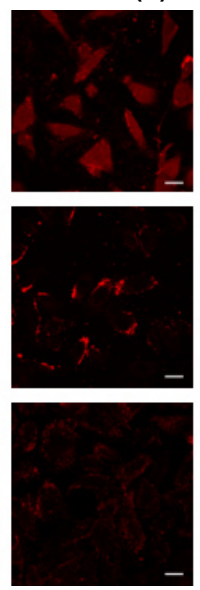

Merged
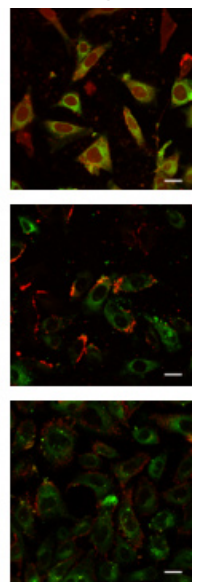

B

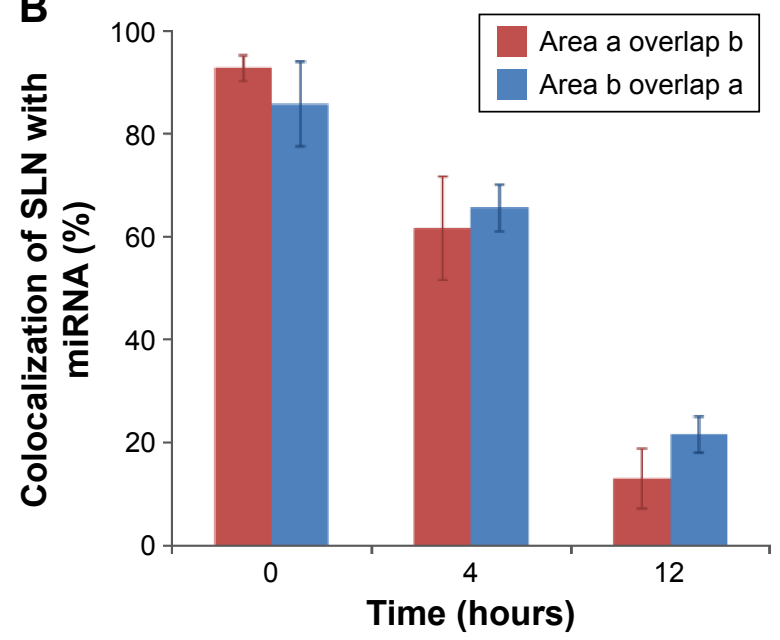

Figure $\mathbf{6}$ Intracellular miRNA release profiles of SLN/miRNA complexes.

Notes: The mammospheres were treated with enzyme to obtain single-layer cells and then incubated with FITC-SLN/miRNA-CY3 for 4 hours. Then, the incubation medium was removed and replaced, and the cells were observed for the next 0, 4, and 12 hours. (A) and (B) represent colocalization micrographs and analysis of SLN with miRNA, respectively. The green color represents SLN, and the red color represents miRNA. Area a (FITC-labeled SLN) overlap b (CY-3-labeled miRNA) represents the proportion of SLN that was occupied by miRNA, and area b overlap a shows the proportion of miRNA that was occupied by SLN. Scale bar: $20 \mu \mathrm{m}$.

Abbreviations: FITC, fluorescein isothiocyanate; SLN, solid lipid nanoparticles.

\section{Cellular uptake of NLC}

The cellular uptakes of fluorescent NLC were viewed by CLSM. Figure 8 shows single section images (A and C) of different depth and 3D images (B and D) reconstructed by piling up the layers of different depths of mammospheres after incubation with fluorescent NLC for 12 and 24 hours, respectively. As shown in Figure 8B and D, obvious fluorescence was observed after 12 hours and the fluorescence intensity was enhanced in 24 hours. The images showed that more FITC-labeled NLC were internalized by mammospheres with incubation for a longer time. At the same depth (Figure 8A and C), the mammosphere presented more fluorescent NLC in the inner cell layers after 24-hour incubation. It illustrated that the NLC went deeper into the mammospheres over time. The results indicated that the cellular uptakes of NLC were time-dependent.
A

TUBB3

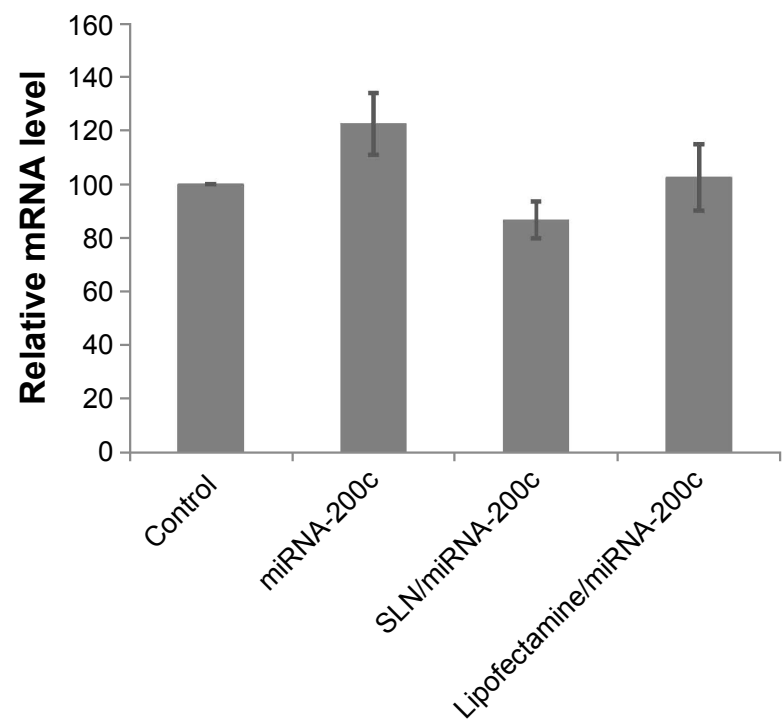

B

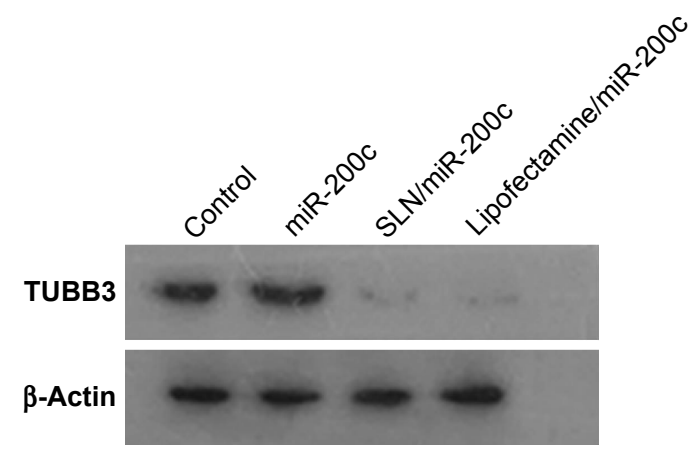

Figure 7 TUBB3 mRNA expression (A) and protein expression (B) after transfection. Abbreviations: SLN, solid lipid nanoparticles; TUBB3, class III beta-tubulin. 


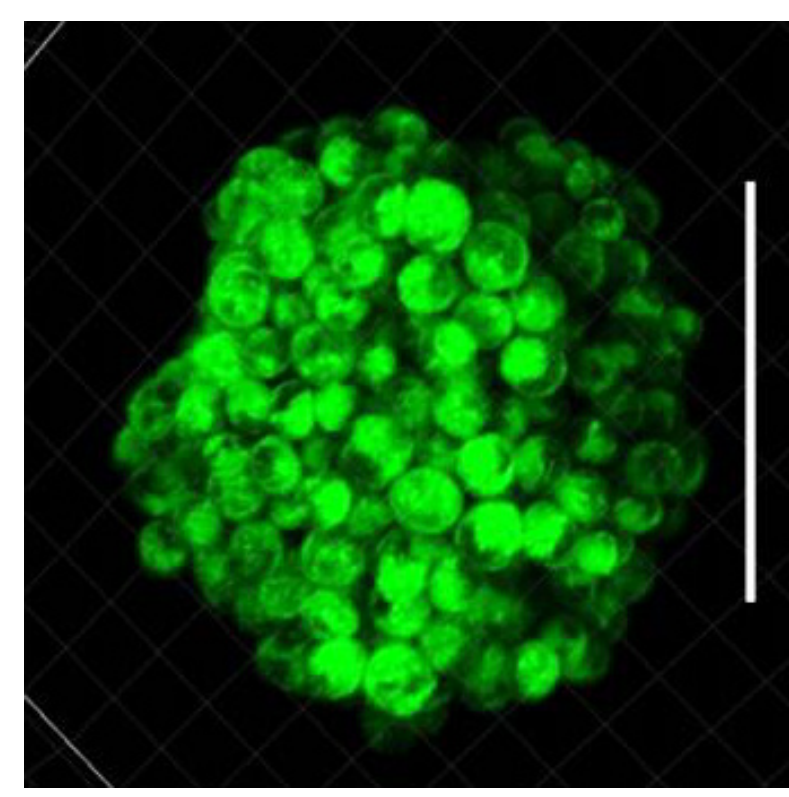

$\boldsymbol{m}$

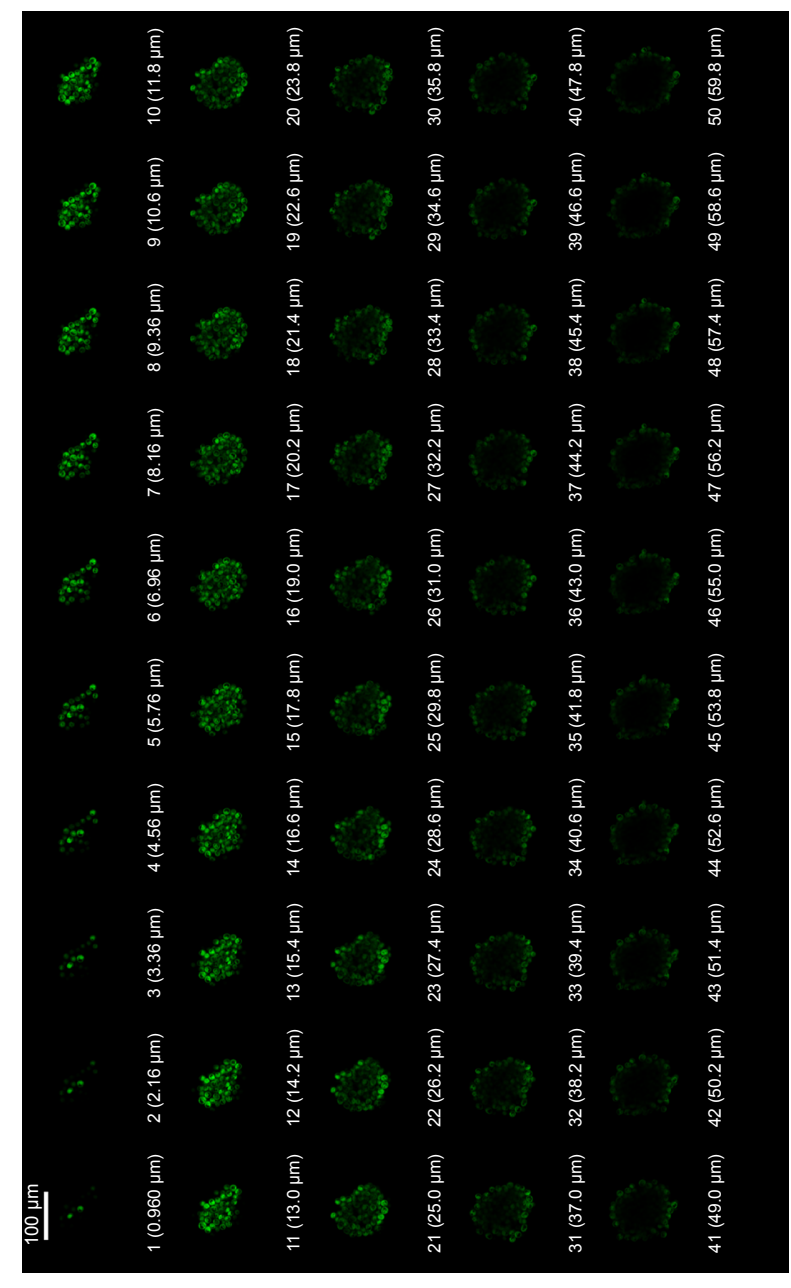

4

Sנnoy Zl

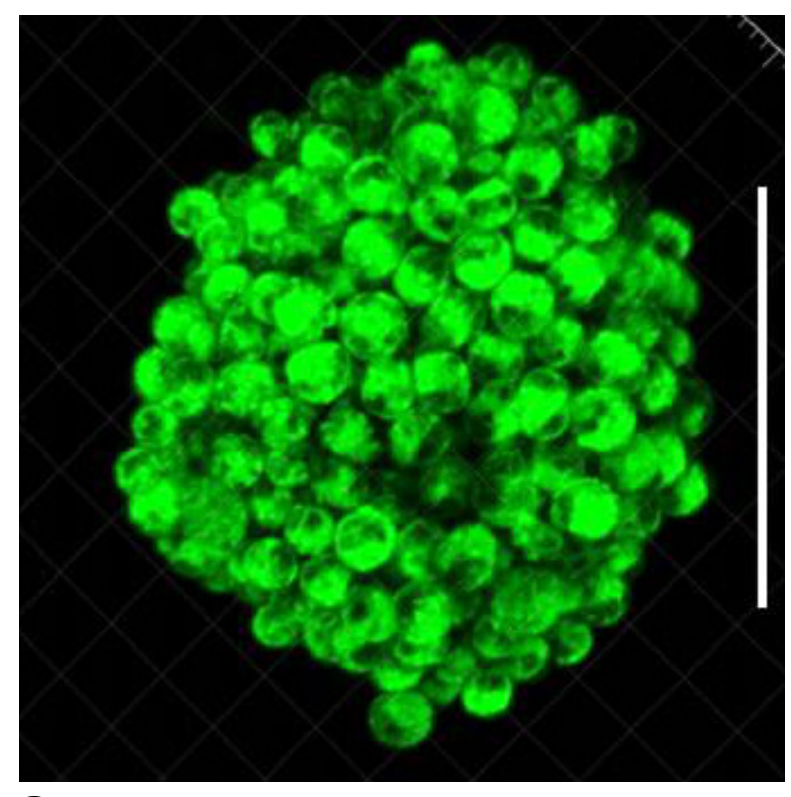

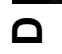

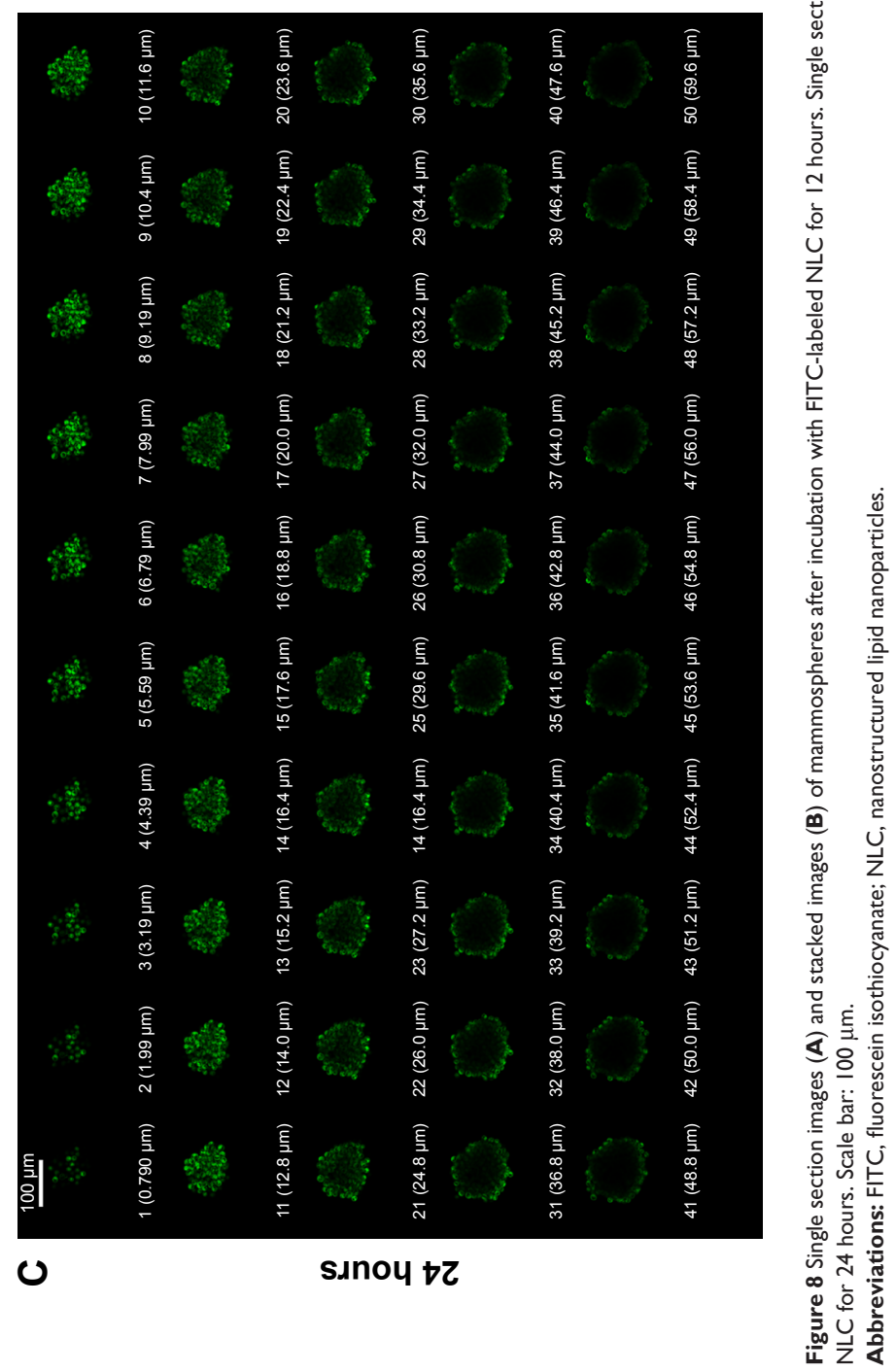


In the meanwhile, confocal microscopy revealed that NLC were localized in cytosol. As the action target of PTX is microtubules in the cytoplasm, the result showed that NLC could deliver PTX into cells and enable localization of PTX to its intracellular site of action.

\section{In vitro anticancer efficacy}

The cytotoxicity is strongly dependent on the cationic lipid used in the matrix lipid. Here, we strategically selected DOTAP to formulate SLN because it is relatively well tolerated compared with other cationic lipids. ${ }^{31}$ The cytotoxicity was evaluated by acid phosphatase assay ${ }^{32}$ and the morphology changes of mammospheres after transfection were observed by an inverted microscope. The cell viabilities of SLN/miRNA NC and Lipofectamine ${ }^{\mathrm{TM}}$ 2000/miRNA NC complexes were $96.42 \%$ and $80.8 \%$, respectively $(* * P<0.01)$ (Figure 9A). As shown in Figure 9B, the influence of SLN/miRNA NC on mammospheres was almost negligible, while the mammospheres treated by Lipofectamine ${ }^{\mathrm{TM}}$ 2000/miR-200c became less compact and regular. The results indicated that SLN were safer gene carriers for miRNA delivery than Lipofectamine ${ }^{\mathrm{TM}} 2000$.

NLC exhibited low cytotoxicity at the concentration of $10-100 \mu \mathrm{g} / \mathrm{mL}$ (Figure 9C). Even when a high concentration

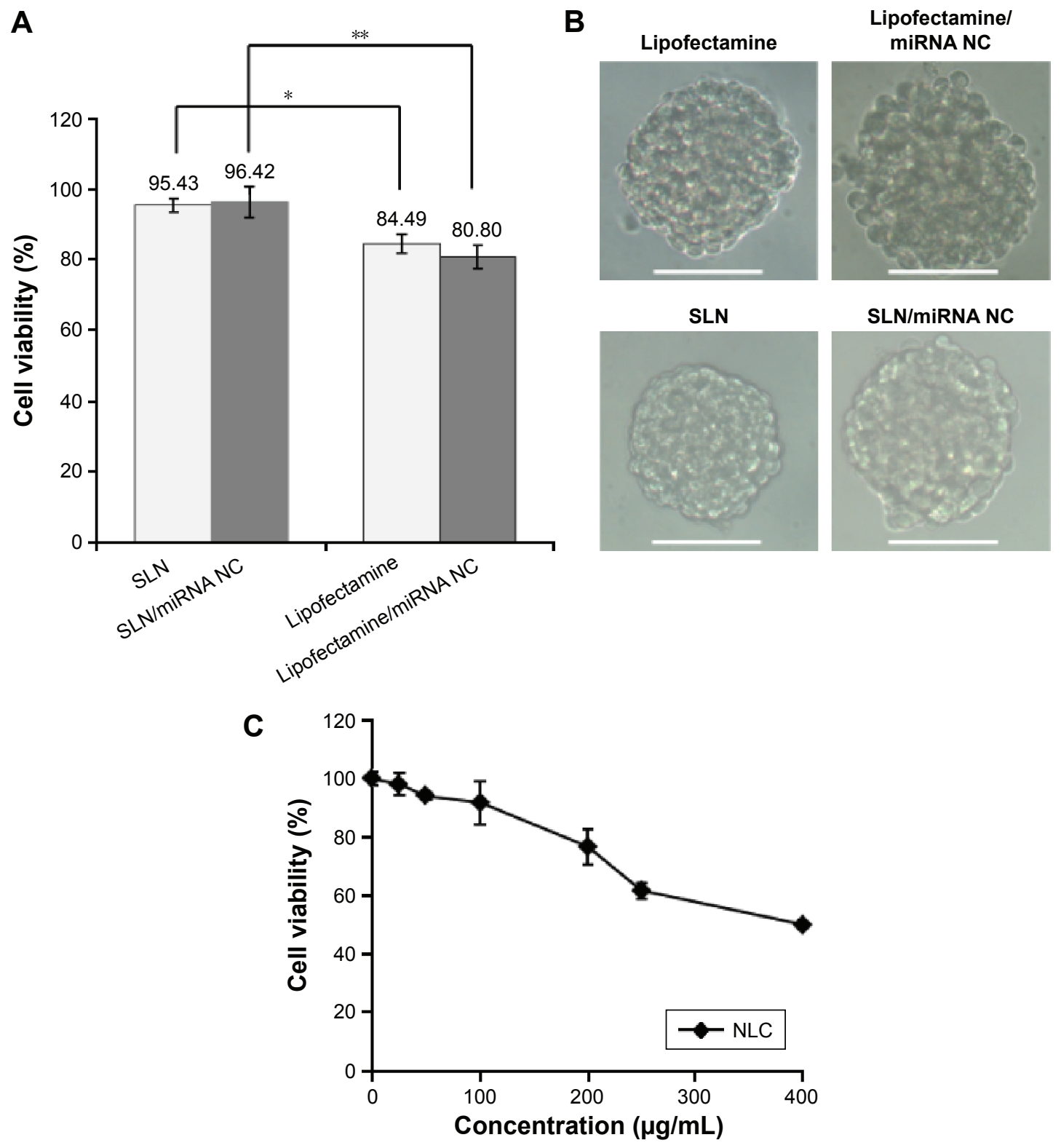

Figure 9 (A) Cell viabilities of SLN, SLN/miRNA NC, Lipofectamine ${ }^{\mathrm{TM}} 2000$, and Lipofectamine ${ }^{\mathrm{TM}}$ 2000/miRNA NC; (B) Light microscope images of mammospheres under different treatments, scale bar: $100 \mu \mathrm{m}$; and (C) Cell viabilities of NLC at various concentrations. $* P<0.05$, $* * P<0.01$.

Abbreviations: NLC, nanostructured lipid nanoparticles; SLN, solid lipid nanoparticles; SLN/miRNA NC, SLN/miRNA negative control. 
was used $(400 \mu \mathrm{g} / \mathrm{mL}), \sim 50 \%$ cells in mammospheres survived, suggesting that NLC could be applied as a safe carrier for chemotherapeutical drug delivery.

It has been reported that restoration of miR-200c could increase the sensitivity to microtubule-targeting chemotherapeutic drugs by reduction of the expression of TUBB3. ${ }^{15}$ To verify whether miR-200c delivered by SLN could enhance the effects of PTX against BCSC, the in vitro anticancer efficacies of NLC/PTX under different transfection conditions were compared. The in vitro anticancer efficacy was defined in terms of the $50 \%$ growth inhibition (half maximal inhibitory concentration $\left[\mathrm{IC}_{50}\right]$ value) based on an acid phosphatase assay.

From Table 3, the $\mathrm{IC}_{50}$ value for taxol after mammospheres transfected with SLN/miR-200c $(1.08 \pm 0.13 \mu \mathrm{g} / \mathrm{mL})$ was significantly lower than that of blank control group $(5.16 \pm 0.23 \mu \mathrm{g} / \mathrm{mL})$, and $\mathrm{IC}_{50}$ value for NLC/PTX after treated with SLN/miR-200c $(0.28 \pm 0.06 \mu \mathrm{g} / \mathrm{mL})$ was also significantly lower than that of the blank control group $(2.06 \pm 0.13 \mu \mathrm{g} / \mathrm{mL})(* * P<0.01)$. These results revealed that miR-200c could act as a sensitizer of PTX when it was delivered by SLN. The $\mathrm{IC}_{50}$ value for taxol after mammospheres incubated with naked miR-200c showed no difference compared with blank control, and no difference was seen between the $\mathrm{IC}_{50}$ values for NLC/PTX of blank group and miR-200c treated group, suggesting that miR-200c could not exert their pharmacological effect without gene carriers.

Under the same transfection conditions, the $\mathrm{IC}_{50}$ values of PTX-loaded NLC were lower than that of taxol. This means the drug internalization into cell could be enhanced by the encapsulation of NLC matrix. The improved cytotoxicity was probably caused from two reasons. One reason is that the internalization of drug into cells was enhanced when the drug was incorporated in NLC, due to the membrane affinity

Table $3 I_{50}$ values of taxol and NLC/PTX against mammospheres after transfection

\begin{tabular}{lll}
\hline Transfection conditions & \multicolumn{1}{l}{$\mathrm{IC}_{50}(\mu \mathrm{g} / \mathrm{mL})$} & \\
\cline { 2 - 3 } & Taxol & NLC/PTX \\
\hline Without transfection & $5.16 \pm 0.23$ & $2.06 \pm 0.13$ \\
SLN/miRNA NC & $4.85 \pm 0.24$ & $1.86 \pm 0.16$ \\
miR-200c & $4.80 \pm 0.28$ & $1.90 \pm 0.16$ \\
Lipofectamine ${ }^{T M} / \mathrm{miR}-200 \mathrm{c}$ & $1.24 \pm 0.15^{* *}$ & $0.29 \pm 0.05^{* *}$ \\
SLN/miR-200c & $1.08 \pm 0.13^{* *}$ & $0.28 \pm 0.06^{* *}$ \\
\hline
\end{tabular}

Notes: $* * P<0.0$ l, compared with blank control group. Data presented as mean \pm standard deviation.

Abbreviations: $I C_{50}$, half maximal inhibitory concentration; NLC, nanostructured lipid nanoparticles; PTX, paclitaxel; SLN, solid lipid nanoparticles; SLN/miRNA NC, SLN/miRNA negative control. of lipid material and nano-scaled size of NLC. The other one is that NLC accumulated in cytoplasm can serve as a drug container releasing the drug continuously.

\section{Conclusion}

In summary, this study reports the development of lipid nanoparticles for separate delivery of miR-200c and PTX. It has been shown that the SLN/miRNA complexes possessed the ability to protect miRNA from degradation and release miRNA effectively after cellular uptake. Consequently, this formulation could sensitize the cytotoxicity of NLC/PTX against BCSC by negatively regulating TUBB3 expression. In addition, the SLN showed a significant less cytotoxicity compared to Lipofectamine ${ }^{\mathrm{TM}}$ 2000. These results suggest that the cationic SLN could serve as a promising carrier for miRNA delivery. In addition, the combination therapy of miR-200c and PTX revealed a novel therapeutic strategy for the treatment of BCSC.

\section{Acknowledgments}

The authors thank the National Natural Science Foundation of China (NSFC No 81273442 81473144) and the Zhejiang Provincial Program for the Cultivation of High-level Innovative Health Talents for their financial support.

\section{Disclosure}

The authors report no conflicts of interest in this work.

\section{References}

1. Torre LA, Bray F, Siegel RL, Ferlay J, Lortet-Tieulent J, Jemal A. Global cancer statistics 2012. CA Cancer J Clin. 2015;65(2):87-108.

2. Ewertz M, Jensen AB. Late effects of breast cancer treatment and potentials for rehabilitation. Acta Oncol. 2011;50(2):187-193.

3. Weaver DL, Ashikaga T, Krag DN, et al. Effect of occult metastases on survival in node-negative breast cancer. N Engl J Med. 2011;364(5): 412-421.

4. Shibata D. Molecular tumor clocks to study the evolution of drug resistance. Mol Pharm. 2011;8(6):2050-2054.

5. Al-Hajj M, Wicha MS, Benito-Hernandez A, Morrison SJ, Clarke MF. Prospective identification of tumorigenic breast cancer cells. Proc Natl Acad Sci U S A. 2003;100(7):3983-3988.

6. Velasco-Velázquez MA, Homsi N, De La Fuente M, Pestell RG. Breast cancer stem cells. Int J Biochem Cell Biol. 2012;44(4):573-577.

7. Kim YJ, Liu Y, Li S, et al. Co-eradication of breast cancer cells and cancer stem cells by cross-linked multilamellar liposomes enhances tumor treatment. Mol Pharm. 2015;12(8): 2811-2822.

8. Dean M, Fojo T, Bates S. Tumour stem cells and drug resistance. Nat Rev Cancer. 2005;5(4):275-284.

9. Easwaran H, Tsai HC, Baylin SB. Cancer epigenetics: tumor heterogeneity, plasticity of stem-like states, and drug resistance. Mol Cell. 2014; 54(5):716-727.

10. Bartel DP. MicroRNAs: target recognition and regulatory functions. Cell. 2009;136(2):215-233.

11. Iorio MV, Croce CM. MicroRNA involvement in human cancer. Carcinogenesis. 2012;33(6):1126-1133. 
12. Liu C, Tang DG. MicroRNA regulation of cancer stem cells. Cancer Res. 2011;71(18):5950-5954.

13. Bao B, Ahmad A, Azmi AS, Ali S, Sarkar FH. Overview of cancer stem cells (CSCs) and mechanisms of their regulation: implications for cancer therapy. Curr Protoc Pharmacol. 2013; Chapter 14:Unit 14.25.

14. Shimono Y, Zabala M, Cho RW, et al. Downregulation of miRNA200c links breast cancer stem cells with normal stem cells. Cell. 2009; 138(3):592-603.

15. Cochrane DR, Spoelstra NS, Howe EN, Nordeen SK, Richer JK. MicroRNA-200c mitigates invasiveness and restores sensitivity to microtubule- targeting chemotherapeutic agents. Mol Cancer Ther. 2009; 8(5):1055-1066.

16. Cochrane DR, Howe EN, Spoelstra NS, et al. Loss of miR-200c: a marker of aggressiveness and chemoresistance in female reproductive cancers. J Oncol. 2010;2010:821717.

17. Wang H, Jiang Y, Peng H, Chen Y, Zhu P, Huang Y. Recent progress in microRNA delivery for cancer therapy by non-viral synthetic vectors. Adv Drug Deliv Rev. 2015;81:142-160.

18. Bravo V, Rosero S, Ricordi C, et al. Instability of miRNA and cDNAs derivatives in RNA preparations. Biochem Biophys Res Commun. 2007; 353(4):1052-1055.

19. Bai M, Shen M, Teng Y, et al. Enhanced therapeutic effect of Adriamycin on multidrug resistant breast cancer by the ABCG2-siRNA loaded polymeric nanoparticles assisted with Ultrasound. Oncotarget. 2015;6(41):43779-43790.

20. Yao Y, Su Z, Liang Y, Zhang N. pH-Sensitive carboxymethyl chitosanmodified cationic liposomes for sorafenib and siRNA co-delivery. Int J Nanomedicine. 2015;10:6185-6197.

21. Yoon HY, Son S, Lee SJ, et al. Glycol chitosan nanoparticles as specialized cancer therapeutic vehicles: sequential delivery of doxorubicin and Bcl-2 siRNA. Sci Rep. 2014;4:6878.

22. Young Yhee J, Son S, Lee H, et al. Nanoparticle-based combination therapy for cancer treatment. Curr Pharm Des. 2015;21(22): 3158-3166.
23. Yoon G, Park JW, Yoon IS. Solid lipid nanoparticles (SLNs) and nanostructured lipid carriers (NLCs): recent advances in drug delivery. $J$ Pharm Invest. 2013;43(5):353-362.

24. He SN, Li YL, Yan JJ, et al. Ternary nanoparticles composed of cationic solid lipid nanoparticles, protamine, and DNA for gene delivery. Int J Nanomedicine. 2013;8:2859-2869.

25. Hu FQ, Zhang Y, Du YZ, Yuan H. Nimodipine loaded lipid nanospheres prepared by solvent diffusion method in a drug saturated aqueous system. Int J Pharm. 2008;348(1):146-152.

26. Morille M, Passirani C, Vonarbourg A, Clavreul A, Benoit JP. Progress in developing cationic vectors for non-viral systemic gene therapy against cancer. Biomaterials. 2008;29(24):3477-3496.

27. Muthiah M, Park IK, Cho CS. Nanoparticle-mediated delivery of therapeutic genes: focus on miRNA therapeutics. Expert Opin Drug Deliv. 2013;10(9):1259-1273.

28. Hu FQ, Jiang SP, Du YZ, et al. Preparation and characterization of stearic acid nanostructured lipid carriers by solvent diffusion method in an aqueous system. Colloids and Surf. B: Biointerfaces. 2005; 45(3):167-173.

29. Dontu G, Abdallah WM, Foley JM, et al. In vitro propagation and transcriptional profiling of human mammary stem/progenitor cells. Genes Dev. 2003;17(10):1253-1270.

30. Wahid F, Shehzad A, Khan T, Kim YY. MicroRNAs: synthesis, mechanism, function, and recent clinical trials. Biochim Biophys Acta. 2010;1803(11):1231-1243.

31. Tabatt K, Sameti M, Olbrich C, Müller RH, Lehr CM. Effect of cationic lipid and matrix lipid composition on solid lipid nanoparticle-mediated gene transfer. Eur J Pharm Biopharm. 2003;57(2):155-162.

32. Friedrich J, Eder W, Castaneda J, et al. A reliable tool to determine cell viability in complex 3 -d culture: the acid phosphatase assay. $J$ Biomol Screen. 2007;12(7):925-937.
International Journal of Nanomedicine

\section{Publish your work in this journal}

The International Journal of Nanomedicine is an international, peerreviewed journal focusing on the application of nanotechnology in diagnostics, therapeutics, and drug delivery systems throughout the biomedical field. This journal is indexed on PubMed Central, MedLine, CAS, SciSearch ${ }^{\circledR}$, Current Contents ${ } /$ Clinical Medicine,

\section{Dovepress}

Journal Citation Reports/Science Edition, EMBase, Scopus and the Elsevier Bibliographic databases. The manuscript management system is completely online and includes a very quick and fair peer-review system, which is all easy to use. Visit http://www.dovepress.com/ testimonials.php to read real quotes from published authors. 